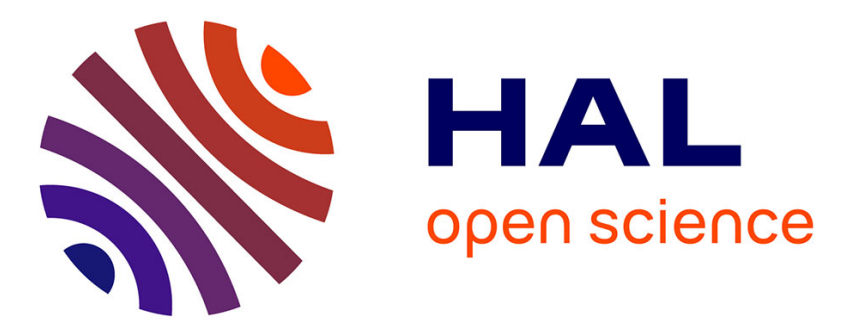

\title{
Testing a bead-rod contact with a nonlinear resonance method
}

\author{
Aurélien Merkel, Georgios Theocharis, F. Allein, J.-P. Groby, Vitali Goussev, \\ Vincent Tournat
}

\section{- To cite this version:}

Aurélien Merkel, Georgios Theocharis, F. Allein, J.-P. Groby, Vitali Goussev, et al.. Testing a beadrod contact with a nonlinear resonance method. Journal of Sound and Vibration, 2019, 441, pp.84-95. 10.1016/j.jsv.2018.10.026 . hal-02325907

\section{HAL Id: hal-02325907 https://hal.science/hal-02325907}

Submitted on 6 Dec 2019

HAL is a multi-disciplinary open access archive for the deposit and dissemination of scientific research documents, whether they are published or not. The documents may come from teaching and research institutions in France or abroad, or from public or private research centers.
L'archive ouverte pluridisciplinaire HAL, est destinée au dépôt et à la diffusion de documents scientifiques de niveau recherche, publiés ou non, émanant des établissements d'enseignement et de recherche français ou étrangers, des laboratoires publics ou privés. 


\title{
Testing a bead-rod contact with a nonlinear resonance method
}

\author{
A. Merkel ${ }^{\mathrm{a}, *}$, G. Theocharis ${ }^{\mathrm{a}}$, F. Allein ${ }^{\mathrm{a}}$, J.-P. Groby ${ }^{\mathrm{a}}, \mathrm{V} . \mathrm{Gusev}^{\mathrm{a}}, \mathrm{V}$. \\ Tournat $^{\mathrm{a}, *}$ \\ ${ }^{a}$ LAUM UMR CNRS 6613, Université du Maine, Av. O. Messiaen, 72085 Le Mans, \\ France.
}

\section{Abstract}

We study the dynamics of an elastic structure composed of a cylindrical rod in contact with a bead at one extremity. Wave propagation within the cylindrical rod is considered linear and dispersionless while the bead-rod contact shows a highly nonlinear behavior as expected from the Hertz's model of contact. The resonance curves of the nonlinear contact depend on the excitation amplitude, where a downshift of the resonance frequency with increasing excitation amplitude is observed. The prediction of the resonance frequency shift by the Hertz's model is compared to the experimental results and shows a disagreement. A better agreement is found by considering the losses with a viscoelastic model, namely the Kuwabara and Kono or Brilliantov model. The observation of the nonlinear effects linked to the resonance of the massspring system can lead to the design of nonlinear elastic metamaterials, where the wave propagation is controlled by nonlinear isolated resonators.

Keywords: Nonlinear resonance, Elastic waves, Granular

\footnotetext{
*Corresponding authors

Email addresses: aurelien.merkel.etu@univ-lemans.fr (A. Merkel ), vincent.tournat@univ-lemans.fr (V. Tournat )
} 
PACS: $43.25 .+\mathrm{y}$

PACS: 45.70.-n

PACS: 46.40.Ff

\section{Introduction}

In studying granular materials, especially when considering the wave dynamics, the definition of the contact law between the particles is of primary importance. The elastic component of the normal contact force between two elastic spheres, without solid bridge between them, is well described in acoustics by the Hertz's contact law [1], which remains relevant in many cases $[1,2]$. In this model, the relation between the force $F$ and the relative displacement between the two sphere centers $\delta$ scales as $F \propto \delta^{3 / 2}$, which represents a highly nonlinear relation that purely comes from the geometry of the problem. In addition to the nonlinear behavior, another consequence of the Hertz's model is the low equivalent moduli of the contact compared to the elastic parameters of the material constituting the bodies in contact. As a result, the velocities of acoustic waves in granular media are small compared to the velocities of acoustic waves propagating in the bulk of elastic solids. The problem of one bead in contact with a homogeneous elastic medium can be viewed, at frequencies much below the first spheroidal resonance of the bead, as the problem of a rigid mass connected to the elastic medium by a nonlinear stiffness. This bead in contact can therefore be considered as a nonlinear isolated resonator since the low equivalent moduli of the contact induces a resonance of this mass-spring system at frequencies where the incoming wavelength in the elastic medium is much larger than the diameter 
of the bead.

The wave propagation within an elastic solid can be controlled by the interaction of the elastic wave with nonlinear isolated resonators, namely elastic spheres in contact. These features have been used to design an acoustic rectifier [3], tunable functional switches [4], tunable phononic crystals [5] or to study the attenuation of surface waves in a colloidal based metamaterial $[6,7]$. Nevertheless, the fine design of a metamaterial based on the nonlinear behavior of granular materials requires a quantitative agreement between the model of contact and the actual experimental behavior.

The comparison of the predictions of the contact models with experimental measurements have essentially been performed by studying the collision process and the coefficient of restitution of a bouncing ball (see [8-12] and the references therein). The problem of collision between two particles has large implications, from astrophysics (Saturn's rings and planet formation for instance) to industrial processes (granulation, handling of fine powders to name a few) and numerous models have been proposed (see [10, 11] and the references therein).

Here, we propose a different approach to study the granular contact restraining ourselves to the case of elastic or viscoelastic contact, which are relevant in the case of acoustic wave propagation (small oscillating or pulsed strains). We consider the case of one bead in contact with a homogeneous elastic solid and which is maintained in contact only by its own weight. This structure presents a resonance frequency and can be considered as a nonlinear isolated elastic resonator. The resonance frequency depends on the amplitude of excitation as it can be seen in Fig. 1(c), where the resonance frequency is 
decreasing with increasing excitation amplitude. It offers the possibility to compare the experimental results of the widely observed nonlinear effect of resonance frequency shift with the predictions of the theoretical models and conclude whether the latter capture correctly this feature. The experimental setup, which is shown in Fig. 1(a) and (b), is reduced to its simplest form and the unknown parameters are therefore limited. The amplitude-dependence of the resonance frequencies of granular media has been previously used to characterize the compaction of a granular assembly [13, 14]. In addition, nonlinear elastic resonances are typical in geomaterials [15], cracked solids and more generally mesoscopic materials containing internal solid contacts $[16,17]$, and for this reason have been also widely studied in the context of ultrasonic non destructive testing [18-21].

This article is organized as follows. In Sec. 2, the experimental setup is described. In Sec. 3, the modeling of the wave propagation in the elastic rod is reviewed. In Sec. 4, the theoretical models to be compared to the experimental results are presented while in Sec. 5, the theoretical predictions of the resonance frequency shift by each contact model are derived. Then, in Sec. 6, the theoretical predictions are compared to the experimental results and finally, in Sec. 7, the obtained results including difficulties arising with the analysis of the $Q$ factors are discussed.

\section{Experimental setup}

The acoustic wave is excited on one side of a cylindrical rod by a piezoelectric transducer. On the other side, the rod is in contact with a spherical bead, which is maintained only by its own weight as shown in Fig. 1(a) and 
(b). The rod is made of stainless steel with a Poisson's ratio of $\nu=0.27$, a mass density $\rho=7.7 .10^{3} \mathrm{~kg} \cdot \mathrm{m}^{-3}$ and a Young's modulus in the range $E=190-210 \mathrm{GPa}$. The bead is also made of stainless steel, and its material parameters are assumed to be the same as those of the rod. The length of the rod is $l_{\text {rod }}=10 \mathrm{~cm}$. The first acoustic longitudinal resonance of the rod is measured at $8.5 \mathrm{kHz}$ (the interface between the rod and the piezoelectric transducer is neither purely rigid nor free, that is why this first resonance cannot be predicted easily with the sound velocity and the length of the rod). The radius of the bead is $r_{\text {bead }}=7.938 \mathrm{~mm}$. The section of the rod in contact with the bead has been polished to enhance the reproducibility of the results. The roughness of the bead is $\sim 25 \mathrm{~nm}$. The dynamic displacement of the bead is measured by a laser vibrometer. The excitation and acquisition are performed using a Stanford SR 785 analyzer in a swept sine mode from 1.5 to $3.5 \mathrm{kHz}$, from low to high frequencies and from high to low frequencies, as excitation signal. The amplitude of the excitation signal follows the protocol shown in Fig. 1(d). The first measurement is performed at very low amplitude of excitation in order to determine the resonance frequency in the linear regime. The resonance frequency found from this first measurement is considered as the reference resonance frequency $f_{0}$ for the protocol. The amplitude of excitation is then increased step by step, and between each step, the measurement at low amplitude is performed again in order to monitor the evolution of the linear resonance frequency $f_{0}(n)$. If the shift $\Delta f_{0}=\left[f_{0}(n)-f_{0}\right] / f_{0}$ of the measured linear resonance frequency compared to the reference frequency is larger than $2 \%$, the following (higher amplitude) measurements of the protocol are removed from the analysis. The 




(b) Laser interferometer
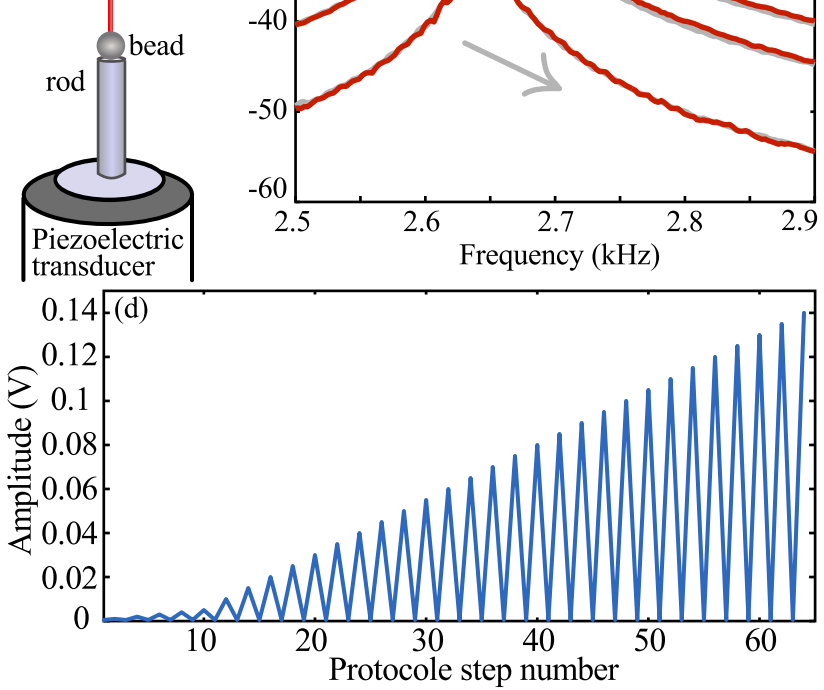
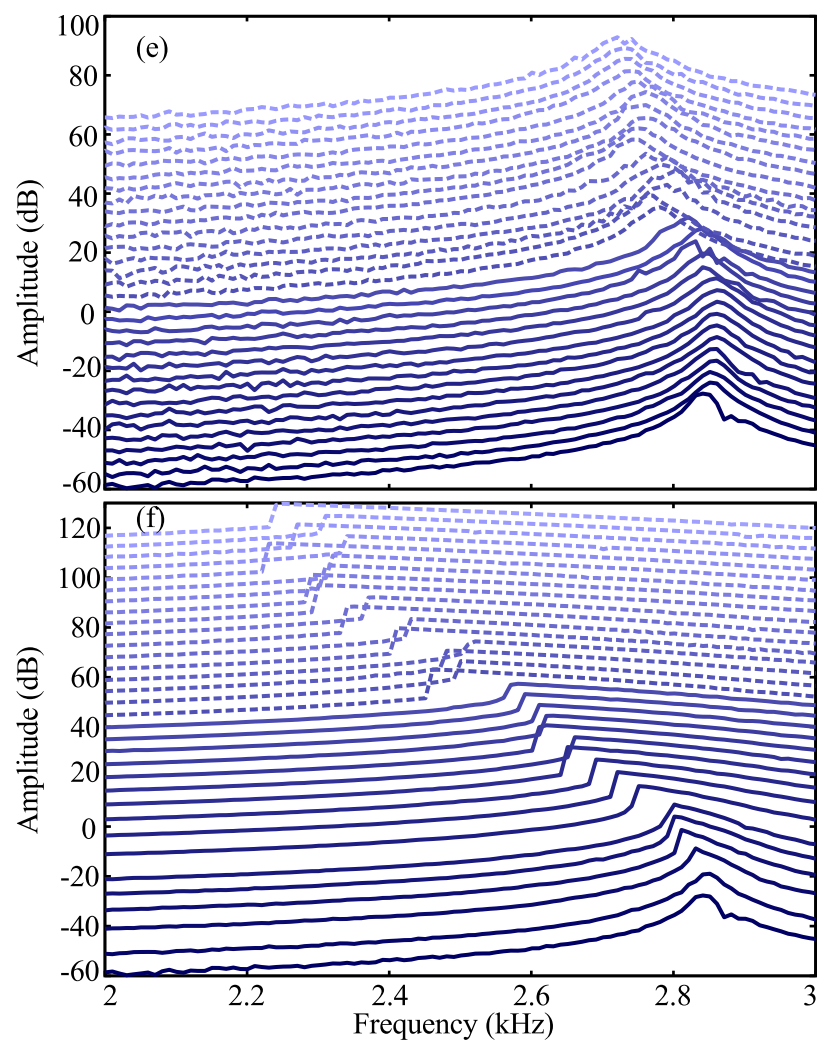

Figure 1: (a) Picture and (b) schematics of the experimental setup. (c) Example of measured displacements of the bead $\delta$ normalized by the static overlap $\delta_{0}$ at different amplitudes of excitation. The resonance frequency depends on the amplitude of excitation and at the larger amplitudes on whether the excitation frequency sweep is performed from low to high frequencies or from high to low frequencies. (d) Experimental protocol of excitation amplitude. Example of one measurement protocol with the resonance frequency (e) in the linear regime and (f) in the nonlinear regime. The dashed curves correspond to the data removed from the analysis.

experimental dataset is composed of 28 amplitude protocols. An example of the results obtained from one measurement protocol is shown in Fig. 1(e) and (f). 


\section{Wave propagation in rods}

The compressional wave propagation takes place in the rod with a diameter $a_{r}=12 \mathrm{~mm}$. The plane wave propagation is assumed when the radius of the rod is much smaller than the wavelength. Thus, the considered frequency range is assumed to be at low frequencies, i.e., $\omega /(2 \pi)<V_{T} /\left(2 \pi a_{r}\right) \simeq 40 \mathrm{kHz}$ where $\omega$ is the cyclic frequency and $V_{T}$ is the transverse wave velocity in the material constituting the rod, and the wave is considered as longitudinal and the propagation unidimensional. The dispersion relations of the longitudinal modes can be found by numerically solving Pochhammer-Chree equation [22]

$2 p\left(q^{2}+k^{2}\right) J_{1}\left(p a_{r}\right) J_{1}\left(q a_{r}\right) / a_{r}-\left(q^{2}-k^{2}\right)^{2} J_{0}\left(p a_{r}\right) J_{1}\left(q a_{r}\right)-4 k^{2} p q J_{1}\left(p a_{r}\right) J_{0}\left(q a_{r}\right)=0$,

where $q^{2}=\omega^{2} / V_{T}^{2}-k^{2}, p^{2}=\omega^{2} / V_{L}^{2}-k^{2}, k$ is the wave number, $J_{n}$ are Bessel function of the first kind and order $n$, and $V_{L}$ is the velocity of longitudinal wave in the material constituting the rod. At low frequencies, the dispersion of the first longitudinal mode remains small and the group and phase velocities $v_{g}$ and $v_{\phi}$, respectively, can be found from the Rayleigh's equation

$$
v_{g} \simeq v_{\phi} \simeq \sqrt{\frac{E}{\rho}}\left[1-\nu\left(\frac{k a_{r}}{2}\right)^{2}\right],
$$

where $E$ is the Young's modulus of the material constituting the rod, $\rho$ and $\nu$, its mass density and its Poisson's ratio, respectively. The group velocity of the first longitudinal mode is estimated to be $v_{g}=4950-5220 \mathrm{~m} \cdot \mathrm{s}^{-1}$ as shown in Fig. 2. 




Figure 2: Group velocity of the first longitudinal mode from the Pochhammer-Chree equation resolution with $E=210 \mathrm{GPa}$ (blue dots) and $E=190 \mathrm{GPa}$ (green dots) and from the Rayleigh's equation with $E=210 \mathrm{GPa}$ (red continuous curve) and $E=190 \mathrm{GPa}$ (orange continuous curve).

\section{Contact models}

\subsection{Hertz model}

Following the Hertz theory of contact $[1,23]$, the relative displacement $\delta$ between two spheres, with a radius $r_{1}$ and $r_{2}$, in contact under a force $F$ is

$$
\delta=\left[\frac{3}{4} \frac{F}{E^{*} R^{1 / 2}}\right]^{2 / 3}, \text { and } F=4 E^{*} R^{1 / 2} \delta^{3 / 2} / 3,
$$

where $R$ is the relative radius of the contact which is deduced from

$$
R=\frac{r_{1} r_{2}}{r_{1}+r_{2}}
$$

and

$$
\frac{1}{E^{*}}=\frac{1-\nu_{1}^{2}}{E_{1}}+\frac{1-\nu_{2}^{2}}{E_{2}},
$$


where $E_{1,2}$ and $\nu_{1,2}$ are the Young's modulus and Poisson's Ratio, respectively, of the beads 1 and 2 . In our case, the bead of radius $r_{1}$ is in contact with a plane surface. This sphere-plane contact can be modeled considering that the radius $r_{2}$ tends to infinity and consequently $R=r_{1}$. The amplitude-dependent normal stiffness $K_{N}$ of the contact is obtained from the force-displacement relation in Eq. (3) with

$$
K_{N}=\partial F / \partial \delta=2 E^{*}(R \delta)^{1 / 2} .
$$

The overlap $\delta$ can be decomposed into a static overlap $\delta_{0}$, resulting here from the applied static force induced by the weight of the bead, and a dynamic overlap $\delta_{d}=\delta_{a} \sin (\tau)$ with $\tau=\omega t$, which is due to the acoustic wave. At the first order of approximation and by considering that the amplitude of the acoustic displacement $\delta_{a}$ is much smaller than the static overlap $\delta_{0}$ i.e., $\delta_{a} \ll \delta_{0}$, the contact between the bead and the rod can be approximated by the normal linear stiffness

$$
K_{0}=\left.\frac{\partial F}{\partial \delta}\right|_{\delta_{0}}=2 E^{*}\left(R \delta_{0}\right)^{1 / 2}
$$

From the normal linear stiffness, the linear resonance frequency of the mass-

spring system can be found for a mass $m_{b}$ of the bead with $f_{0}=\left(K_{0} / m_{b}\right)^{1 / 2} /(2 \pi)$. The static external force $F_{0}$ applied on the contact is only due to the weight of the bead, no additional normal force is introduced. The static overlap, linear stiffness and linear resonance frequency are predicted to be, considering the two values of Young's Modulus $E=190 \mathrm{GPa}$ and $E=210 \mathrm{GPa}$, $\delta_{0}=55-50 \mathrm{~nm}, K_{0}=4.3 \cdot 10^{6}-4.6 \cdot 10^{6} \mathrm{~N} \cdot \mathrm{m}^{-1}$ and $f_{\text {res }}^{\text {Hertz }}=2.60-2.70 \mathrm{kHz}$. 


\subsection{Viscoelastic model}

In the numerous studies on the collision between two particles, dissipative forces are added to the purely elastic contact model from Hertz. Dissipation at the contact level can come from different mechanisms that contribute to the energy losses. The first one is the radiation of acoustic waves in the two bodies. The second one is the possible plastic deformation. The third one is the losses due to the viscoelastic behavior of the material constituting the beads [24]. Here, we consider only the last type of dissipative mechanism by neglecting the acoustic energy radiation and assuming that there is no plastic deformation at the contact $[24,25]$. Considering a yield stress of $Y=6.12 \mathrm{GPa}$ for stainless steel, the limit force for plastic deformations can be estimated to be $F_{l}=1.6^{3} \pi^{3} Y^{3} R^{2} /\left(24 E^{* 2}\right) \simeq 6.5 .10^{3} \mathrm{~N}$ [2], which is much larger than estimated dynamic maximum dynamic force at $3 \mathrm{kHz}$ $F_{d}=m_{b} \omega^{2} \delta_{0} \simeq 0.3 \mathrm{~N}$, wich of the same as the static force $F_{0} \simeq 0.16 \mathrm{~N}$ applied here.

Considering all the possible models of viscoelastic contact between two spheres, we use here the model developed in [24-26], where a viscous stress proportional to the strain rate is introduced [27] into the analysis of the contact problem. The viscoelastic description corresponds therefore to a Kelvin-Voigt model. The dissipation due to viscoelasticity adds a dissipative part in the normal force which is written $[25,26]$

$$
F=\frac{4}{3} E^{*} R^{1 / 2}\left(\delta^{3 / 2}+\frac{3}{2} A \delta^{1 / 2} \frac{\partial \delta}{\partial t}\right),
$$

with

$$
A=\frac{1}{E} \frac{1+\nu}{1-\nu}\left[\frac{4}{3} \eta_{1}\left(1-\nu+\nu^{2}\right)+\eta_{2}(1-2 \nu)^{2}\right]
$$


and where $\eta_{1,2}$ are the bulk viscosities of the material constituting the beads, made of the same material with Young's modulus $E$ and Poisson's ratio $\nu$. Due to the lack of information on the dissipative coefficients $\eta_{1,2}$, the viscous bulk constants are considered as fitting parameters with $\eta_{1}=\eta_{2} \equiv \eta$. The definition of the parameter $A$ can change following the references. The one given in [25] has been corrected in [28] and has been recalculated in [26]. It should be noticed that a similar expression to Eq. (8) can be obtained by considering an imaginary part in the elastic moduli, thus writing the normal force as

$$
F=\left(C_{1}+i C_{2}\right) \delta^{3 / 2}=\left(C_{1}+i \omega C_{2}^{\prime}\right) \delta^{3 / 2}=C_{1} \delta^{3 / 2}+C_{2}^{\prime} \delta^{1 / 2} \frac{\partial \delta}{\partial t},
$$

where $C_{1}, C_{2}$ and $C_{2}^{\prime}$ are constants. The geometry of the problem of the contact between two spheres induces, in addition to the nonlinear forcedisplacement relation, a displacement-dependent dissipative force. It should be noticed that this model is in agreement with the experimental measurements of the restitution coefficient $e_{n}$ and its dependence on the impact velocity $v_{0}$. By writing the elastic force in the general form [9]

$$
f^{e l} \propto \delta^{1+\alpha}
$$

and the viscoelastic force in the general form

$$
f^{v i s} \propto \frac{\partial \delta}{\partial t} \delta^{\xi_{0}}\left(\frac{\partial \delta / \partial t}{\partial \delta_{0} / \partial t}\right)^{\xi_{1}}
$$

where $\partial \delta_{0} / \partial t$ is a typical velocity scale, the velocity dependence of the restitution coefficient is then [9]

$$
1-e_{n} \propto v_{0}^{\left[2\left(\xi_{0}+\xi_{1}\right)-\alpha\left(1-\xi_{1}\right)\right] /(2+\alpha)} .
$$


In order to get a good agreement with the experimental results [8, 9], the exponent in Eq. (13) should be equal to $1 / 5$ i.e.,

$$
\frac{2\left(\xi_{0}+\xi_{1}\right)-\alpha\left(1-\xi_{1}\right)}{2+\alpha}=\frac{1}{5}
$$

which is the case for the viscoelastic model in Eq. (8).

\subsection{Adhesion}

At the contact level, an adhesion can appear due to the interatomic forces. Many models have been developed, among which are the Dejarguin-MullerToporov model (DMT) [29], the Johnson-Kendall-Robert model (JKR) [30] and the Maugis-Dugdale model (MD) [31] ; each of them being relevant depending on the problem under study. The prevalence of one of the model can be determined with the adhesion map of Johnson et al. [32]. The adhesion map is governed by two parameters. The first one is the Muller's parameter $\mu$ defined with $[33,34]$

$$
\mu=\frac{32}{3 \pi z_{0}}\left(\frac{\gamma^{2} R}{E^{* 2}}\right)^{1 / 3},
$$

where $\gamma$ is the surface energy, $z_{0}$ is the equilibrium separation. Similarly, the Tabor's parameter $\lambda[35]$ can be used and is defined as $\lambda=1.16 \mu$. It should be noticed that the work of adhesion $w$ (commonly denoted as surface energies $\left.\Delta \gamma=\gamma_{1}+\gamma_{2}+\gamma_{12}\right)$ is often used instead of the the surface energy $\gamma$. When the two contact surfaces are made of the same material, we have $w=2 \gamma$. The second parameter is the ratio of the external applied force to the adhesion force. If the external applied force $F$ is large enough compared to the adhesion force $F_{a}$ i.e., $F_{a} / F<0.05$, then the Hertz model can be applied [32]. Considering a small external force compared to the adhesion 
force and if $\mu<1$, then the DMT model applies. If $\mu>5$, then the JKR model applies. If $1<\mu<5$, then the MD model applies.

Here, considering the surface energy of stainless steel as $\gamma=0.7 \mathrm{~J}^{-2}$ and the equilibrium separation $z_{0}=4 \cdot 10^{-10} \mathrm{~m}[36], \mu=57$ and the JKR model applies since the static external force $F_{0}$ is small $F_{0} /(2 \pi \gamma R)=4.5$. The adhesion force (pull-off force) is then $F_{c}=3 \pi \gamma R$. The relation between the external force $F$ and the relative displacement $\delta$ is not explicit but is implicit through the contact radius $a$. The contact radius is controled by $[34]$

$$
a^{3}=\frac{3 R}{4 E^{*}}\left[F+2+F_{c}+\left(4 F F_{c}+4 F_{c}\right)^{1 / 2}\right] .
$$

The relative displacement $\delta$ and the force $F$ are

$$
\delta=\frac{\pi a}{2 E^{*}}\left(p_{0}+2 p_{0}^{\prime}\right) \text { and } F=\pi a^{2}\left(\frac{2 p_{0}}{3}+2 p_{0}^{\prime}\right),
$$

with

$$
p_{0}=\frac{2 a E^{*}}{\pi R} \text { and } p_{0}^{\prime}=-\left(\frac{4 \gamma E^{*}}{\pi a}\right)^{1 / 2} .
$$

The linear stiffness of the contact is written [34]

$$
K_{0}^{j k r}=2 E^{*} a\left[3-3\left(a_{c} / a\right)^{3 / 2}\right] /\left[3-\left(a_{c} / a\right)^{3 / 2}\right],
$$

where $a_{c}^{3}=3 F_{c} R /\left(4 E^{*}\right)$. The static relative displacement predicted by the JKR model is $\delta_{0}=67-62 \mathrm{~nm}$, the resonance frequency is $f_{\text {res }}^{j k r}=2.74-$ $2.86 \mathrm{kHz}$. The force displacement relation of the Hertz and JKR model are plotted in Fig. 3.

\subsection{Roughness}

The surface roughness of the beads can also affect the force-displacement relation. By considering the contact between two spheres with fractal rough 


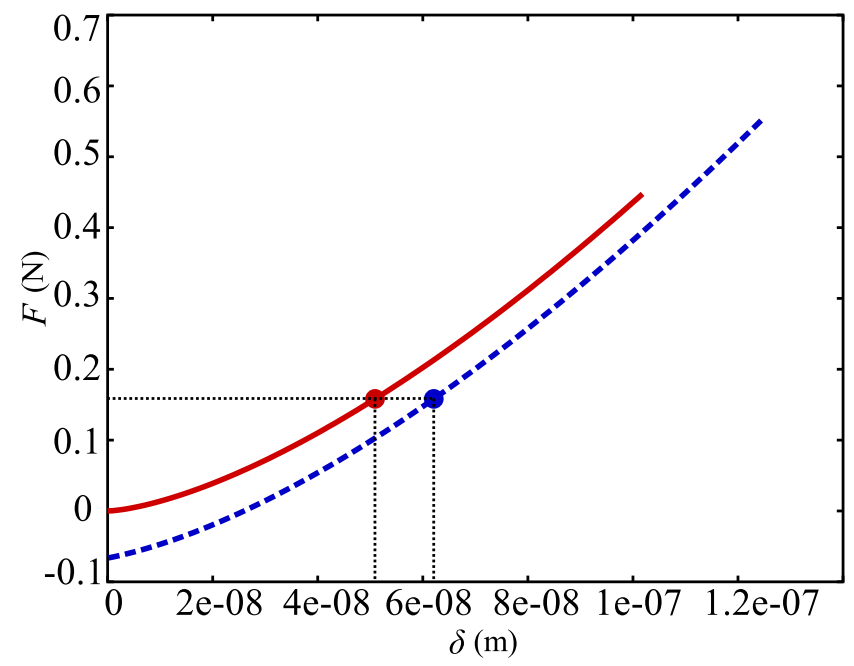

Figure 3: Force-overlap $F-\delta$ relation from the Hertz model (red curve) and the JKR model (blue dashed curve). The static overlaps are marked by circle for both models.

surfaces, the following relation between the stiffness and the applied force is found [37]:

$$
K_{N} \propto F^{1 /(H+1)},
$$

where $H(0<H \leq 2)$ is the Hurst exponent that characterizes the roughness of the surfaces. For $H=2$, the Hertz law is found. This leads to a forcedisplacement relation of the form

$$
F \propto \delta^{n}=\delta^{(1+H) / H} .
$$

Therefore, $3 / 2 \leq n<+\infty$ depending on the value of $H$. Following another approach, previous studies indicate that the values of the exponent $n$ can be modified by the presence of an oxide layer, whose elastic modulus is much lower than the one of the initial material, at the surface of the sphere and forms a soft shell. The soft shell model gives $n=2$ [38]. 


\section{Resonance frequency shift}

Using the Hertz or JKR models, the linear resonance frequency is predicted to be below $3 \mathrm{kHz}$, which is far below the first resonance of the rod measured at $f_{0}^{r o d} \simeq 8.5 \mathrm{kHz}$. This experimental system can therefore be reduced to the simplest case of single mass-spring system, the mass being the mass of the bead $m_{b}$, the spring being the contact. As stated before, the stiffness of the contact is found by deriving the nonlinear force-displacement relation $K_{N}=\partial F / \partial \delta$ and thus depends on the overlap $\delta$, which can be decomposed into a static part $\delta_{0}$ and a dynamic part due to the acoustic wave $\delta_{a} \sin (\tau)$, giving $\delta=\delta_{0}+\delta_{a} \sin (\tau)$. At very low amplitude of excitation, the acoustic amplitude $\delta_{a}$ is negligible against the static overlap $\delta_{0}$. The resonance frequency only depends on the static part of the overlap.

When the acoustic amplitude becomes non-negligible against the static overlap, the resonance frequency depends on the amplitude of excitation. The resonance frequency $f^{r e s}$ is proportional to the square root of the ratio of the stiffness $K_{N}$ of the contact to the mass $m_{b}$, i.e., $f^{r e s}=\left(K_{N} / m_{b}\right)^{1 / 2} /(2 \pi)$. Then, at the first order, $\Delta f / f_{0} \simeq\left(\Delta K_{N} / K_{0}-\Delta m / m_{b}\right) / 2$. Since the mass of the bead $m_{b}$ is constant in the case here, the resonance frequency shift $\Delta f / f_{0}$ is only proportional to the relative modification of the stiffness $\Delta f / f_{0} \simeq \Delta K_{N} /\left(2 K_{0}\right)$. The relative modification of the stiffness $\Delta K_{N}$ can be found by averaging the stiffness $K_{N}$ over a wave period, which is written $\Delta K_{N} / K_{0}=\left\langle K_{N}-K_{0}\right\rangle / K_{0}=\left\langle\partial F / \partial \delta-K_{0}\right\rangle / K_{0}$. Finally, we obtain $\Delta f / f_{0} \simeq\left\langle\partial F / \partial \delta-K_{0}\right\rangle /\left(2 K_{0}\right)[13,39]$. As shown in Fig. 4, the first order approximation of the resonance frequency shift remains valid for all the cases considered here. This procedure can be applied to all the contact models ex- 
posed in Sec. 4 .

\subsection{Hertz model}

Considering the force-displacement relation in Eq. (3), we find that

$$
\frac{\Delta K_{N}-K_{0}}{K_{0}}=\frac{2 E^{*}(R \delta)^{1 / 2}-2 E^{*}\left(R \delta_{0}\right)^{1 / 2}}{2 E^{*}\left(R \delta_{0}\right)^{1 / 2}}=\left(\frac{\delta_{a} \sin \tau+\delta_{0}}{\delta_{0}}\right)^{1 / 2}-1 .
$$

Finally, the resonance frequency shift predicted by the Hertz model is found with

$$
\frac{\Delta f^{h e r t z}}{f_{0}} \simeq \frac{1}{4 \pi} \int_{0}^{2 \pi}\left(\frac{\delta_{a}}{\delta_{0}} \sin \tau+1\right)^{1 / 2} d \tau-\frac{1}{2}
$$

which can be solved numerically.

\subsection{Viscoelastic contact}

Considering the force-displacement relation in Eq. (8), the stiffness $K_{N}$ of the contact is

$K_{N}=\frac{\partial F\left(\delta_{0}+\delta_{a} \sin \tau\right)}{\partial \delta}=\frac{\partial\left\{\frac{4}{3} E^{*} \sqrt{R}\left[\left(\delta_{0}+\delta_{a} \sin \tau\right)^{3 / 2}+\frac{3}{2} A \omega\left(\delta_{0}+\delta_{a} \sin \tau\right)^{1 / 2} \delta_{a} \cos \tau\right]\right\}}{\partial \delta_{a} \sin \tau}$,

where $\omega$ is the cyclic pulsation at the resonance. Finally, the resonance frequency shift predicted by the viscoelastic model is

$$
\frac{\Delta f^{v i s}}{f_{0}} \simeq \frac{1}{4 \pi} \int_{0}^{2 \pi}\left(\frac{\delta_{a}}{\delta_{0}} \sin \tau+1\right)^{1 / 2} d \tau+\frac{A}{4 \pi \delta_{0}^{1 / 2}} \int_{0}^{2 \pi} \frac{\partial\left(\omega\left(\delta_{0}+\delta_{a} \sin \tau\right)^{1 / 2} \delta_{a} \cos \tau\right)}{\partial \delta_{a} \sin \tau} d \tau-\frac{1}{2},
$$

which can also be solved numerically. Because of the nonlinear behavior of the viscoelastic losses induced by the geometry of the problem (contact sphereplane), the viscoelastic losses have an influence on the resonance frequency shift, which is represented by an additional term in Eq. (25) compared to the prediction in Eq. (23). 


\subsection{Adhesion}

Since there is no explicit relation between the force and the displacement

in the JKR model, the prediction of the resonance frequency shift has to be performed numerically.

The resonance frequency shift predicted by the Hertz, viscoelastic and JKR models are shown in Fig. 4 as a function of the overlap $\delta_{a} / \delta_{0}$. The predictions from the viscoelastic model present a large resonance frequency shift while the predictions from the Hertz and JKR models do not substantially differ. The JKR model will therefore not be considered in the comparison with the experimental measurement of the resonance frequency shift.

\subsection{Surface roughness}

Considering the force-displacement relation in Eq. (21), the stiffness is

$$
K_{N}=\frac{\partial F}{\partial \delta}=n C\left(\delta_{0}+\delta\right)^{n-1}
$$

where $C$ is a constant. The resonance frequency shift is then predicted with

$$
\frac{\Delta f}{f_{0}} \simeq \frac{1}{4 \pi} \int_{0}^{2 \pi}\left(\frac{\delta_{a}}{\delta_{0}} \sin t+1\right)^{n-1} d t-\frac{1}{2} .
$$

The maximum frequency shift for $\delta_{a}=\delta_{0}$ as a function of the exponent $n$ is shown in Fig. 5. The frequency shift is negative if $1<n<2$, and is positive otherwise. The minimum value predicted in this case of resonance frequency shift is around -0.05 for a value of the exponent close to the one of the Hertz model $n=3 / 2$. As seen in Fig. 1(c), the resonance frequency is decreasing with increasing excitation amplitude. This indicates that $1<n<2$ and that this model can predict a resonance frequency shift similar or smaller than the one predicted by the Hertz model. 


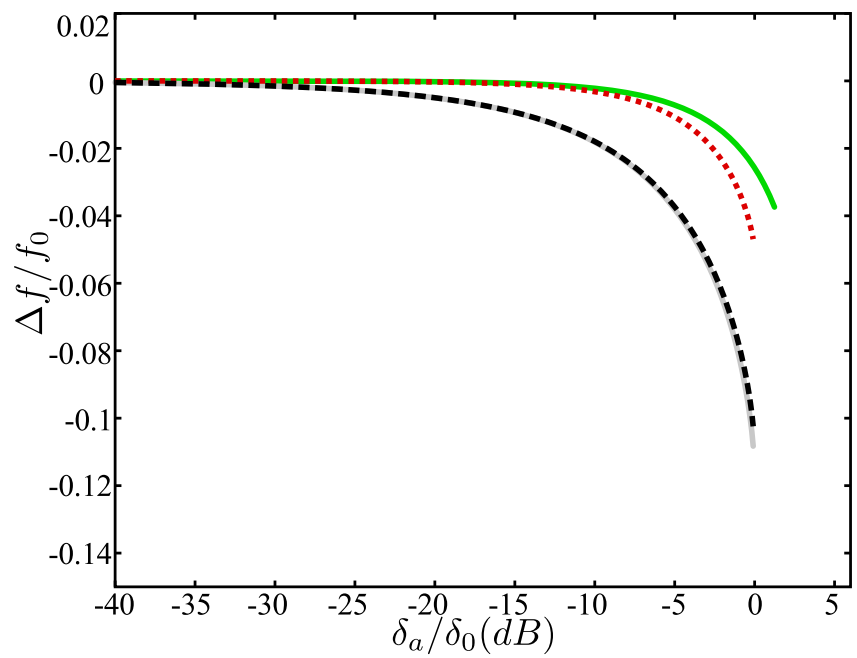

Figure 4: Theoretical predictions of the resonance frequency shift $\Delta f / f_{0}$ as a function of the acoustic overlap $\delta_{a}$ from the Hertz (red dotted curve), JKR (green continuous curve) and viscoelastic with $\eta=1.05 \mathrm{MPa} . \mathrm{s}^{-1}$ (black dashed curve) models. Theoretical predictions of the resonance frequency shift without the first order approximation, i.e., $\Delta f / f_{0}=\left(\sqrt{K_{N} / m_{b}}-\sqrt{K_{0} / m_{b}}\right) / \sqrt{K_{0} / m_{b}}$ from the viscoelastic model (gray continuous curve). The acoustic overlap $\delta_{a}$ is normalized to the static overlap $\delta_{0}$ predicted by the Hertz model.

\section{Experimental results}

The bead and the rod are assumed to be composed of the same material, the dissipative constant is chosen to be $\eta=1.05 \mathrm{MPa} \cdot \mathrm{s}^{-1}$ giving $A=1 \cdot 1 \cdot 10^{-5}$. The Young modulus is $E=210 \mathrm{GPa}$ in the following analysis. The measured linear resonance frequencies $f_{0}$ of each of performed protocols can be compared to the predictions of the Hertz and the JKR models $f_{0}^{\text {Hertz }}$ and $f_{0}^{J K R}$, which is shown in Fig. 6. The range of experimentally measured linear resonance frequencies is larger than the range of the combined predictions of both Hertz and JKR models. This is attributed to the irregularity of 


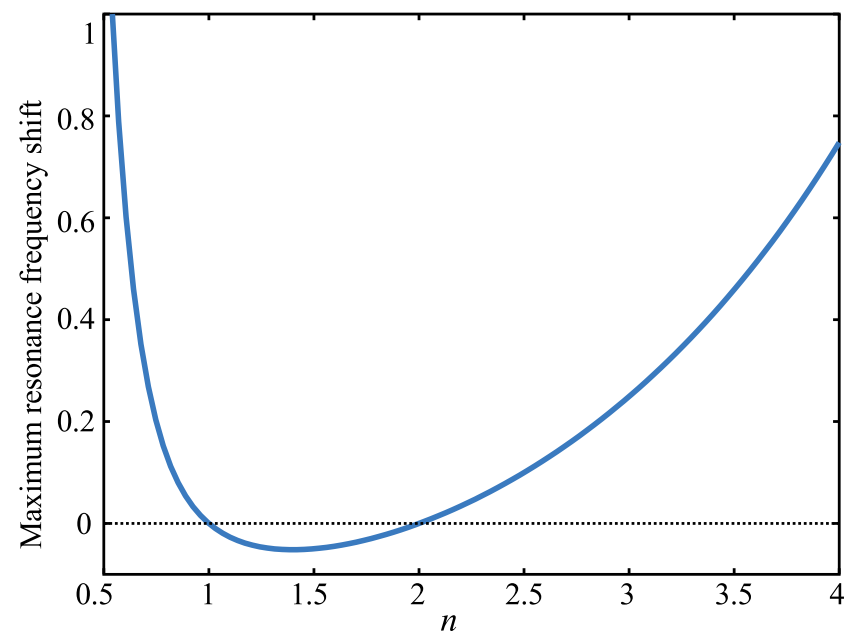

Figure 5: Maximum resonance frequency shift for $\delta_{a}=\delta_{0}$ as a function of the exponent of the force-displacement relation predicted from Eq. (27).

the surfaces in contact. For instance, the rugosity of bead surface is $25 \mathrm{~nm}$ (manufacturer's data), which is not negligible compared to the static overlap predicted, $\delta_{0}=55-67 \mathrm{~nm}$. In conclusion, these experimental results do not allow us to discriminate between those two models.

In Fig. 7, the experimental measurements of the resonance frequency shift are compared to the theoretical predictions from the Hertz and the viscoelastic models of contact. The Hertz model, as well as the JKR model and the model including the surface roughness following the conclusions of Sec. 5, underestimate the resonance frequency shift. It should be noticed that there is no fitting parameter in the Hertz model in Eq. (23) that could allow us to find a good agreement between the Hertz model and the experimental results. In Fig. 7 , the $x$-coordinate is normalized by $\delta_{0}$, which is the only 


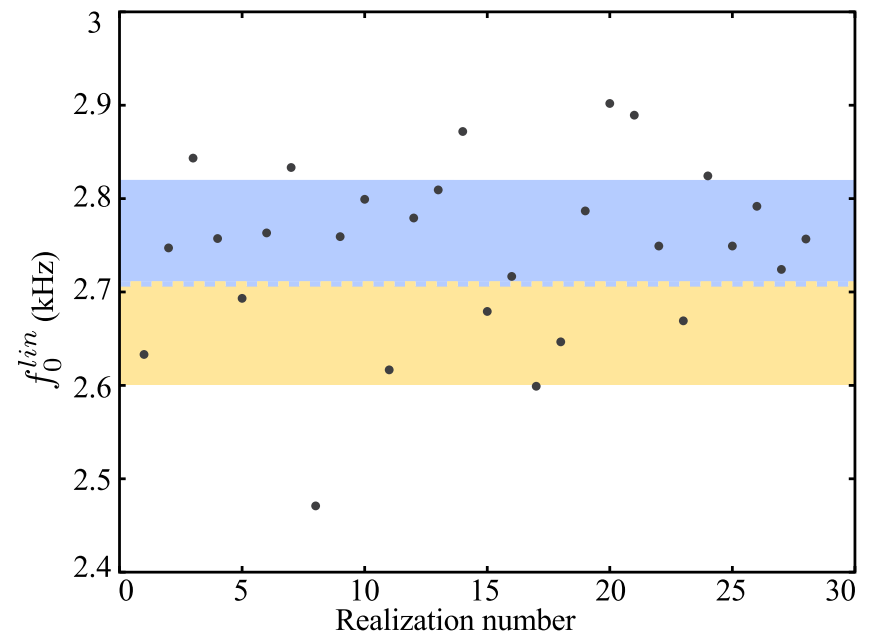

Figure 6: Comparison between the experimental linear resonance frequencies and the predictions from Hertz (yellow area) and JKR (blue area) models

contributing parameter in Eq. (23). Moreover, the disagreement between the Hertz model can also be seen on the slope of the frequency shift with the displacement amplitude in the right panel in Fig. 7. The main contribution of the Hertz model shows a dependence proportional to $\delta_{a}^{2}$ [39], whereas the experimental results show a dependence closer to $\delta_{a}^{3 / 2}$, which is impossible to achieve using the Hertz model. On the other hand, the viscoelastic model shows a good agreement with the experimental results.

\section{Discussion on the damping parameters of the viscoelastic model}

\subsection{Comparison with previous studies}

The estimation of the parameters in this paper are based on the Brilliantov model $[25,26]$, which is similar to the Kuwabara-Kono model, which is written as [24]

$$
F^{k k}=k_{n} \delta^{3 / 2}+\gamma_{n} \delta^{1 / 2} \frac{\partial \delta}{\partial t}
$$



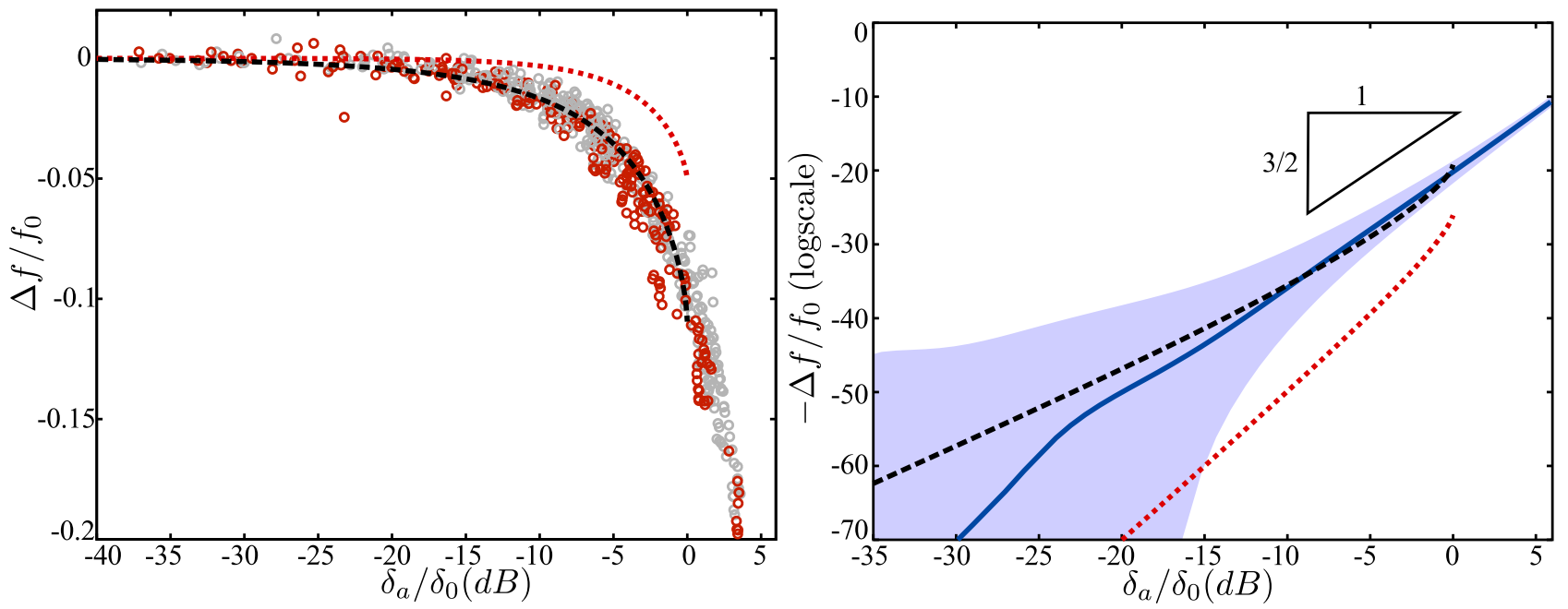

Figure 7: Resonance frequency shift $\Delta f / f_{0}$ in linear scale (left) and in logscale (right) as a function of the normalized acoustic amplitude $\delta_{a} / \delta_{0}$ in logscale. The acoustic ampitude $\delta_{a}$ is the displacement measured at the top of the bead at the resonance. The static displacement $\delta_{0}$ is found from the Hertz theory considering the weight of the bead. The theoretical resonance frequency shifts are predicted from the Hertz model $\Delta f^{H e r t z} / f_{0}$ (red dotted curve) and from the viscolelastic model $\Delta f^{v i s} / f_{0}$ (black dashed curve). (Left) The gray dots correspond to the measurements with increasing frequencies for the sweep. The orange dots correspond to the measurements with decreasing frequencies for the sweep. (Right) Mean value of the frequency shift (blue continuous curve) and interval of $90 \%$ of confidence (blue area).

The Kuwabara-Kono model has been used in [10], where the constants $k_{n}$ and $\gamma_{n}$ have been scaled to fit previous experimental measurement of the impact restitution coefficient between two beads. In [10], the material constants for the stainless steel bead experiments are $E=193 \mathrm{GPa}, \nu=0.35, \rho=$ $7830 \mathrm{~kg} \cdot \mathrm{m}^{-3}$ and the radii of the beads are $r_{1}=r_{2}=1.27 \mathrm{~cm}$. The stiffness constant $k_{n}$ is found from $k_{n}=4 E^{*} R^{1 / 2} / 3=1.17 .10^{10}$ and the fit gives the value $\gamma_{n}=3.31 .10^{4} \mathrm{~kg} \cdot \mathrm{m}^{-1 / 2} \cdot \mathrm{s}^{-1}$. By using the material and geometric 
constants given in [10] and by using $\eta=1.05$. MPa.s ${ }^{-1}$ with Eqs. (8) and (9), we obtain $\gamma_{n}=7.8 .10^{4} \mathrm{~kg} \cdot \mathrm{m}^{-1 / 2} \cdot \mathrm{s}^{-1}$, which is of the same order as the value given in [10].

\subsection{Q factor analysis}

Using the force-diplacement relation in Eq.(8) and keeping only the linear contributions, the linear equation of motion of the bead is in the from

$$
m_{b} \frac{\partial^{2} \delta_{d}}{\partial t^{2}}+2 E^{*} A\left(R \delta_{0}\right)^{1 / 2} \frac{\partial \delta_{d}}{\partial t}+2 E^{*}\left(R \delta_{0}\right)^{1 / 2} \delta_{d}=0
$$

The $Q$ factor of the linear oscillator is then

$$
Q=\omega_{0} m_{b} /\left[2 E^{*} A\left(R \delta_{0}\right)^{1 / 2}\right]
$$

with $\eta=1.05$ Mpa.s $^{-1}, Q=5$. At small amplitude, the measured $Q$ factor of the reference resonance curves are $20<Q<100$ as shown in Fig. 8. It should be noticed that no correlation appears between the resonance frequencies shown in Fig. 6 and the measured $Q$ factors for the same resonance curves as it can be seen in Fig. 8. In Fig. 9, the experimental resonance frequency shift is compared to those predicted with $\eta=0.3 \mathrm{MPa}^{-1}{ }^{-1}$, giving $Q=20$ and $\eta=55 \mathrm{kPa}^{-1}{ }^{-1}$, giving $Q=100$. By decreasing the value of $\eta$, the effect of the viscous losses on the resonance frequency shift decreases as expected. For $Q=100$, the predicted resonance frequency shift is close to the one predicted by the Hertz model. It is important to note that, in the experimental results, the resonance frequency shift does not depend on the $Q$ factor of the reference curves as it can be seen in Fig. 7, where all the measurements present the same behavior. 


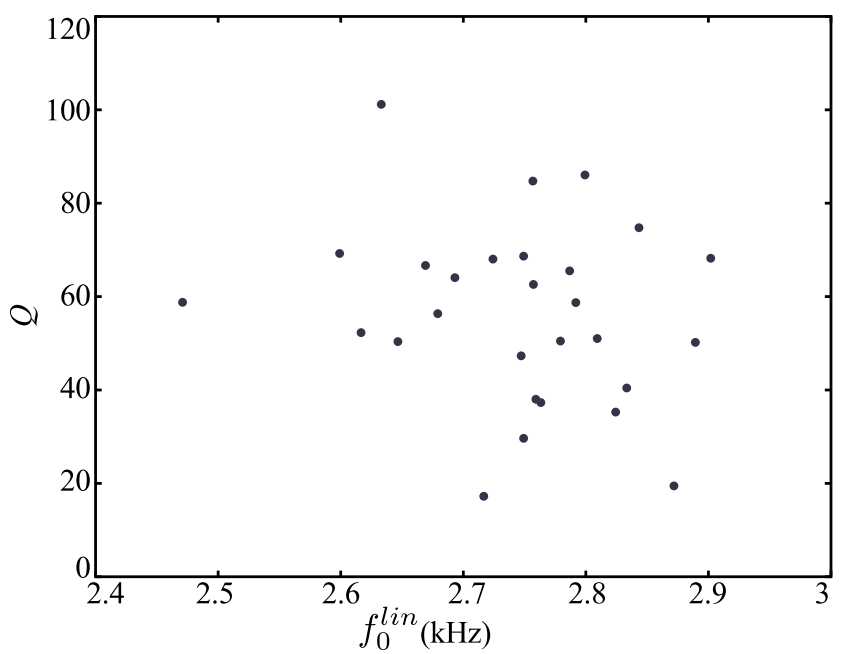

Figure 8: $Q$ factors of the linear resonance curves as a function of $f_{0}^{l i n}$

The evolution of the $Q$ factor with the excitation amplitude cannot be estimated precisely by measuring the width of the resonance curve at half amplitude (or $-3 \mathrm{~dB}$ method) when the resonance curve becomes asymmetric. The amplitude-dependence of the inverse $Q$ factor $\Delta(1 / Q)$ can be found from the relation $[16]$

$$
\Delta\left(\frac{1}{Q}\right)=\frac{1}{Q_{r e f}}\left(\frac{V \delta_{r e f}}{V_{r e f} \delta}-1\right) .
$$

where $Q_{r e f}$ is the $Q$ factor of the reference curve, $V$ are the excitation voltage amplitudes $\left(V_{\text {ref }}\right.$ being the voltage excitation amplitude of the reference measurement), $\delta$ is the displacement amplitude measured at the resonance corresponding to the excitation voltage amplitude $V$ and $\delta_{r e f}$ is the displacement amplitude measured at the resonance corresponding to the excitation voltage amplitude $V_{\text {ref }}$. It can be seen in Fig. 10 that the inverse $Q$ factors do not show a noticeable dependence on the amplitude below amplitudes of displacement of the same order as $\delta_{0}$. 


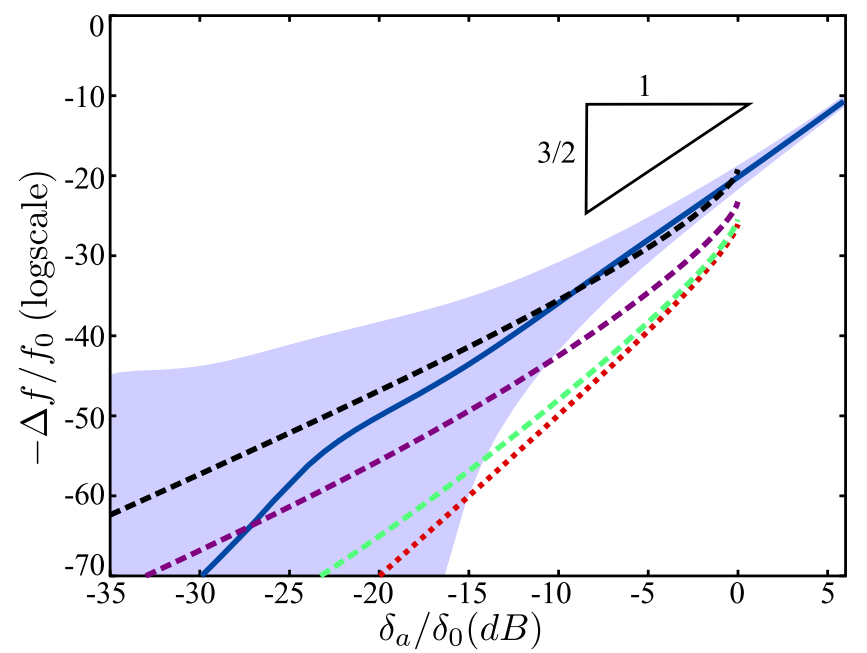

Figure 9: Resonance frequency shift $\Delta f / f_{0}$ in logscale as a function of the normalized acoustic amplitude $\delta_{a} / \delta_{0}$ in logscale. The acoustic ampitude $\delta_{a}$ is the displacement measured at the top of the bead at the resonance. The static displacement $\delta_{0}$ is found from the Hertz theory considering the weight of the bead. Frequency shift predicted from the Hertz model $\Delta f^{H e r t z} / f_{0}$ (red dotted curve) and from the viscolelastic model $\Delta f^{\text {vis }} / f_{0}$ with $Q=5$ (black dashed curve), $Q=20$ (violet dashed curve) and $Q=100$ (green dashed curve). Mean value of the frequency shift (blue continuous curve) and interval of confidence (blue area).

In conclusion of this section, the analysis shows us that the viscoelastic model cannot capture entirely the experimental results. It is not possible to obtain an agreement on both the $Q$ factor of the linear resonances and the nonlinear resonance frequency shift with the same viscous constants. This observation highlights the necessity to refine the model for the viscoelastic losses. In the same manner, the absence of correlation between the linear resonance frequency and the $Q$ factor as well as the dispersion in the measured $Q$ factor values indicate that there are additional dependencies for the $Q$ factor. 
These latter could come from the roughness of the surfaces or the humidity of the surrounding air, leading to an additional layer of viscous fluid at the surface of the bead. For instance, it has already been observed that the presence of a viscous fluid at the surface of the beads induces a hardening of the contacts and increases the dissipation [40,41]. A control of the humidity in the surrounding air seems relevant for future experiments in order to test this assumption.

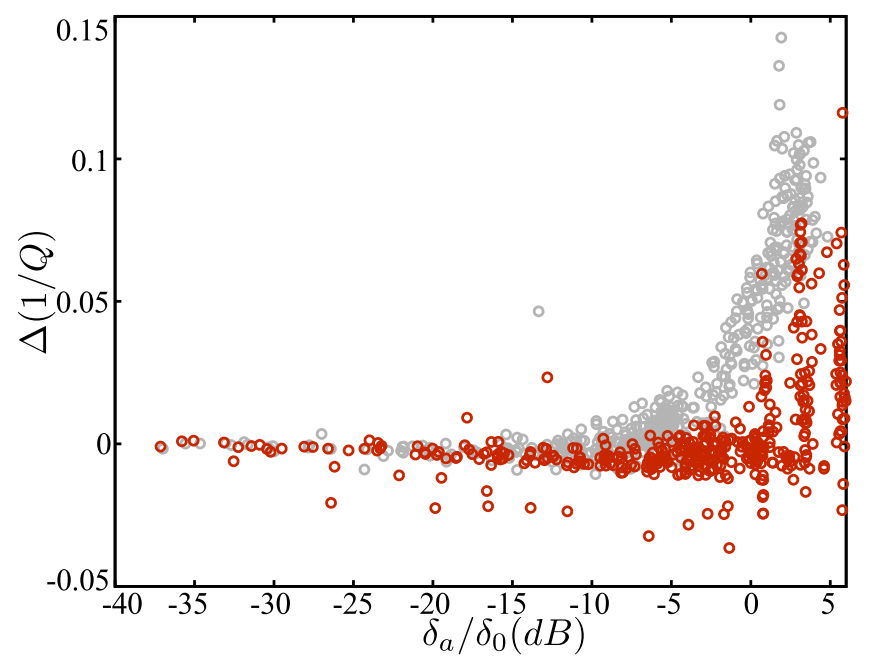

Figure 10: Inverse $Q$ factor shift $\Delta 1 / Q$ in linear scale as a function of the normalized acoustic amplitude $\delta / \delta_{0}$ in logscale. The gray dots correspond to the measurements with increasing frequencies for the sweep. The orange dots correspond to the measurements with decreasing frequencies for the sweep.

\section{Summary}

In this study, one bead in contact with an elastic solid is considered as a nonlinear isolated elastic resonator. The nonlinear effect of resonance frequency shift, i.e., amplitude-dependent resonance frequency, is analyzed 
and the experimental results are compared to the theoretical predictions of various contact models. The main conclusion is that the classical Hertzian model is unable to capture the experimental nonlinear behavior as well as the JKR model with adhesion and a model including the roughness of the surfaces in contact. A model accounting for the viscoelastic losses at the level of the contact, which are amplitude-dependent due to the geometry of the contact, is able to fit the experimental results in a more relevant manner. However, the analysis and comparison between the theoretically predicted and experimentally measured $Q$ factors reveal a strong unexpected disagreement. Interestingly, we found that the nonlinear resonance frequency shift is a robust feature of the bead-rod contact problem unlike the linear $Q$ factor and even the linear resonance frequency. This study is a necessary step towards the design of granular-based metamaterials for the purpose of elastic wave control. We believe this study could also guide future research for the investigation of the dynamics of inter-grain contacts with losses.

\section{Acknowledgements}

The authors would like to acknowledge Stefan Luding and Vanessa Magnanimo for fruitful discussions.

The work has been supported by the grant CIG FP7 Comgransol, the grant PROPASYM - Pari scientifique Pays-de-la-Loire and the RFI Le Mans Acoustique (Région Pays de la Loire) PavNat project.

[1] K. L. Johnson, Contact Mechanics, Cambridge University Press, Cambridge, England, 1985. 
[2] C. Coste, B. Gilles, On the validity of hertz contact law for granular material acoustics, Eur. Phys. J. B 7 (1999) 155-168.

[3] N. Boechler, G. Theocharis, C. Daraio, Bifurcation-based acoustic switching and rectification, Nat. Mater. 10 (2011) 665-668.

[4] R. Ganesh, S. Gonella, From modal mixing to tunable functional switches in nonlinear phononic crystals, Phys. Rev. Lett. 114 (2015) 054302 .

[5] F. Allein, V. Tournat, V. Gusev, G. Theocharis, Tunable magnetogranular phononic crystals, Appl. Phys. Lett. 108 (2016) 161903.

[6] A. Geslain, S. Raetz, M. Hiraiwa, M. A. Ghanem, S. P. Wallen, A. Khanolkar, N. Boechler, J. Laurent, C. Prada, A. Duclos, P. Leclaire, J.-P. Groby, Spatial laplace transform for complex wavenumber recovery and its application to the analysis of attenuation in acoustic systems, J. Appl. Phys. 120 (2016) 135107.

[7] M. Hiraiwa, M. A. Ghanem, S. P. Wallen, A. Khanolkar, A. A. Maznev, N. Boechler, Complex contact-based dynamics of microsphere monolayers revealed by resonant attenuation of surface acoustic waves, Phys. Rev. Lett. 116 (2016) 198001.

[8] J. Schäfer, S. Dippel, D. E. Wolf, Force schemes in simulations of granular media, J. Phys. I France 6 (1996) 5-20.

[9] S. Luding, Collisions \& contacts between two particles, in: H. J. Hermann, J.-P. Hovi, S. Luding (Eds.), Physics of Dry Granular Media, Springer, Netherlands, 1998. 
[10] H. Kruggel-Emden, E. Simsek, S. Rickelt, S. Wirtz, V. Scherer, Review and extension of normal force models for the discrete element method, Powder Tech. 171 (2007) 157-173.

[11] A. Singh, V. Magnanimo, S. Luding, A contact model for sticking of adhesive meso-particles, Arxiv (1503.03720).

[12] M. Heckel, A. Glielmo, N. Gunkelmann, T. Pöschel, Can we obtain the coefficient of restitution from the sound of a bouncing ball?, Phys. Rev. E 93 (2016) 032901.

[13] C. Inserra, V. Tournat, V. Gusev, Characterization of granular compaction by nonlinear acoustic resonance method, Appl. Phys. Lett. 92 (2008) 191916.

[14] J.-B. Legland, V. Tournat, O. Dazel, A. Novak, V. Gusev, Linear and nonlinear biot waves in a noncohesive granular medium slab: Transfer function, self-action, second harmonic generation, J. Acoust. Soc. Am. 131 (2012) 4292-4303.

[15] J. A. Tencate, D. Pasqualini, S. Habib, K. Heitmann, D. Higdon, P. A. Johnson, Nonlinear and nonequilibrium dynamics in geomateria, Phys. Rev. Lett. 93 (2004) 065501.

[16] P. Johnson, A. Sutin, Slow dynamics and anomalous nonlinear fast dynamics in diverse solids, J. Acoust. Soc. Am. 117 (2005) 124-130.

[17] R. A. Guyer, P. A. Johnson, Nonlinear Mesoscopic Elasticity: The Complex Behaviour of Rocks, Soil, Concrete, Wiley-VCH Verlag, Weinheim, 2009. 
[18] K. V. D. Abeele, J. de Visscher, Damage assessment in reinforced concrete using spectral and temporal nonlinear vibration techniques, Cement and Concrete Research 30 (2000) 1453-1464.

[19] V. Y. Zaitsev, V. Gusev, B. Castagnède, Luxemburg-gorky effect retooled for the elastic waves: a mechanism and experimental evidence, Phys. Rev. Lett. 89 (2002) 105502.

[20] A. Moussatov, V. Gusev, B. Castagnède, Self-induced hysteresis for nonlinear acoustic waves in cracked material, Phys. Rev. Lett. 90 (2003) 124301.

[21] L. Fillinger, V. Y. Zaitsev, V. E. Gusev, B. Castagnède, Self-modulation of acoustic waves in resonant bars, J. Sound Vib. 318 (2008) 527-548.

[22] D. Royer, E. Dieulesaint, Elastic Waves in Solids I: Free and Guided Propagation, Springer-Verlag, Berlin, Heidelberg, 2000.

[23] J. Duffy, R. D. Mindlin, Stress-strain relations and vibrations of a granular medium, Tech. Rep. No. CU-TR-17., Colunbia Univ. New York Dept. of Civil Engineering and Engineering Mechanics (1956).

[24] G. Kuwabara, K. Kono, Restitution coefficient in a collision between two spheres, Jap. J. Appl. Phys. 26 (1987) 1230-1233.

[25] N. V. Brilliantov, F. Spahn, J.-M. Hertzsch, T. Pöschel, Model for collision in granular gases, Phys. Rev. E 53 (1996) 5382-5392.

[26] N. V. Brilliantov, A. V. Pimenova, D. S. Goldobin, A dissipative force 
between cooliding viscoelastic bodies: Rigorous approach, EPL 109 (2015) 14005.

[27] L. Landau, E. Lifchitz, Theory of Elasticity, Pergamon Press, London, 1970.

[28] Q. J. Zheng, H. P. Zhu, A. B. Yu, Finite element analysis of the contact forces between a viscoelastic sphere and a rigid plane, Powder Tech. 225 (2012) 130-142.

[29] B. V. Dejarguin, V. M. Muller, Y. P. Toporov, Effect of contact deformations on the adhesion of particles, J. Colloid Interface Sci. 53 (1975) 314.

[30] K. L. Johnson, K. Kendall, A. D. Roberts, Surface energy and the contact of elastic solids, Proc. R. Soc. Lond. A 324 (1971) 301-313.

[31] D. Maugis, Adhesion of spheres: The JKR-DMT transition using a Dugdale model, J. Colloid Interface Sci. 150 (1992) 243-269.

[32] K. L. Johnson, J. A. Greenwood, An adhesion map for the contact of elastic spheres, J. Colloid Interface Sci. 192 (1997) 326-333.

[33] V. M. Muller, V. S. Yushenko, B. V. Dejarguin, On the influence of molecular forces on the deformation of an elastic sphere and its sticking to a rigid plane, J. Colloid Interface Sci. 77 (1980) 91.

[34] C. Thornton, K. K. Yin, Impact of elastic spheres with and without adhesion, Powder Tech. 65 (1991) 153-166. 
[35] D. Tabor, Surface forces and surface interactions, J. Colloid Interface Sci. 58 (1977) 2-13.

[36] R. M. Brach, P. F. Dunn, Macrodynamics of microparticles, Aerosol Sci. Tech. 23 (1995) 51-71.

[37] R. Pohrt, V. L. Popov, Contact mechanics of rough spheres: Crossover from fractal to hertzian behavior, Adv. Trib. 2013 (2013) 974178.

[38] P.-G. de Gennes, Static compression of a granular medium: the "soft shell" model, Europhys. Lett. 35 (1996) 145-149.

[39] J. Cabaret, V. Tournat, P. Béquin, Amplitude-dependent phononic processes in a diatomic granular chain in the weakly nonlinear regime, Phys. Rev. E 86 (2012) 041305.

[40] S. Job, F. Santibanez, F. Tapia, F. Melo, Nonlinear waves in dry and wet hertzian granular chains, Ultrasonics 48 (2008) 506-514.

[41] T. Brunet, X. Jia, P. Mills, Mechanism for acoustic absorption in dry and wet granular media, Phys. Rev. Lett. 101 (2008) 138001. 